\title{
RIESGOS DE ORIGEN TECNOLÓGICO: APUNTES CONCEPTUALES PARA UNA DEFINICIÓN, CARACTERIZACIÓN Y RECONOCIMIENTO DE LAS PERSPECTIVAS DE ESTUDIO DEL RIESGO TECNOLÓGICO
}

\author{
Omar Javier Ramírez \\ ECAPMA - Universidad Nacional Abierta y a Distancia $^{1}$
}

Manizales, 2009-11-08 (Rev. 2009-12-04)

\begin{abstract}
RESUMEN
Desde la mirada de las llamadas ciencias naturales y sociales, el estudio del concepto "riesgo" ha estado tradicionalmente ligado a las potenciales amenazas e impactos generados por múltiples eventos de origen natural. No obstante, en los últimos años ha emergido un campo de análisis de los riesgos asociados a la inserción y el desarrollo de eventos tecnológicos (industria química, telecomunicaciones, fuentes energéticas y alimenticias, e incluso el mismo escenario armamentista) aportando con ello relevantes elementos de análisis para pensar la compleja relación entre sociedad y ambiente, en un momento donde el progreso tecnocientífico representa concomitantemente el ascenso de una "sociedad del riesgo". La noción de riesgo tecnológico, así, toma importancia para los análisis contemporáneos, razón por la cual es necesario ahondar en su significado, reconocer las múltiples propuestas conceptuales esgrimidas al respecto y caracterizar las perspectivas académicas desde las cuales se puede avanzar en el estudio del mismo. Sobre estos aspectos transcurre el presente artículo.
\end{abstract}

\section{PALABRAS CLAVE}

Riesgo tecnológico, sociedad del riesgo, tecnología, ciencia, sociedad y ambiente.

\section{TECHNOLOGICAL RISKS: CONCEPTUAL NOTES FOR THE DEFINITION, CHARACTERIZATION AND ACKNOWLEDGEMENT OF STUDY PERSPECTIVES REGARDING TECHNOLOGICAL RISKS}

\begin{abstract}
From the perspective of nNatural and sSocial sSciences, the study of the concept of "risk" concept has been traditionally linked to the potential threats and impacts generated by multiple events of natural origin. However, a field of analysis associated with the introduction and development of technological events (chemical industry, telecommunications, energetic and food sources, even the weapons scenario) has emerged in the last few years. This has provided important elements of analysis elements to think about the complex relation between society and environment in at a time where the techno-scientific progress represents the rise of a "risk society". Thus, the notion of technological risk gains relevance for contemporary analysis, and for this reason it is necessary to delve into its meaning, acknowledge the multiple conceptual proposals that it has created,put forward in this regard and characterize the academic perspectives that can be used from which to advance in theits study. of the mentioned subject. The present article approaches these issues.
\end{abstract}

KEY WORDS: Technological risks, risk society, technology, science, society and environment.

\section{INTRODUCCIÓN}

El campo de reflexión en torno a la relación sociedad y ambiente es sin duda uno de los escenarios que más ha llamado la atención y las miradas de disímiles áreas del conocimiento en los últimos años. Muestra de ello es el conjunto de producción intelectual materializada en libros, revistas, videos, portales de Internet, programas académicos y memorias de encuentros locales, regionales, nacionales e internacionales que versan sobre la denominada problemática ambiental, donde se discuten desde asuntos concernientes a eventos macroecológicos (como el cambio climático y la pérdida de biodiversidad) hasta conflictos ambientales resultados de eventos microecológicos (escenarios de contaminación ambiental local). 
Quizá este aparente auge no sea fortuito y de alguna manera responda al llamado que desde las ciencias naturales y sociales (particularmente desde esta última) se ha realizado para abordar desde marcos holísticos e interdisciplinarios el tema ambiental. Lo anterior no por simple capricho, sino como exigencia necesaria tras el reconocimiento tanto de la complejidad de los acontecimientos enfrentados, como de las limitaciones epistemológicas, metodológicas e instrumentales aparejadas a la aplicación de modelos lineales y reduccionistas al ámbito de estudio de la problemática ambiental (Carrizosa, 2001; Gallopín et al, 2001).

Dentro de este conjunto de perspectivas analíticas de la relación sociedad y ambiente se puede diferenciar una en particular que, en los últimos, ha emergido y avanzado tomando como elemento central de estudio el concepto de "riesgo". Éste, que habitualmente es asociado al campo de estudio de amenazas "naturales" tales como sismos, remoción de masas, inundaciones, entre otros, ha logrado extender su campo de acción hacia el estudio de las amenazas de origen tecnológico que, como resultado del acelerado ritmo tecnocientífico ${ }^{2}$ característico de nuestro tiempo, enfrenta la sociedad actual. Por ello, los riesgos en los que se centra el presente artículo son aquellos originados de eventos tecnológicos (riesgos tecnológicos), los cuales, como se verá más adelante, son analizados tanto desde el punto de vista de perspectivas socio-interpretativas, como desde miradas estadístico-probabilísticas.

Es así que los avances tecnológicos en el ámbito productivo, industrial, químico, energético, alimentario y de comunicaciones, son ahora objeto de análisis, pues su inserción en el entramado social no trae consigo únicamente un conjunto de beneficios, sino también una serie de riesgos -e incluso incertidumbres- que, de materializarse, pueden desencadenar efectos adversos sobre la salud humana y el ambiente en general. Tal es así, que algunos autores han decidido caracterizar el momento actual bajo la denominación de la "sociedad del riesgo" (Beck, 1998; 2002).

Sin embargo, ¿qué se entiende por riesgo? Más exactamente, ¿qué se entiende por riesgo tecnológico? ¿Cómo se interpreta este concepto que ha llamado la atención de legos y expertos? ¿Qué elementos componen la noción de riesgo tecnológico? ¿Qué relevancia mantiene el estudio de este concepto en el mundo contemporáneo?

El presente artículo pretende avanzar conforme a los interrogantes planteados, tratando de destacar la importancia y complejidad que ha alcanzado el debate en torno al concepto en mención, lo cual se ve plasmado en la pluralidad de enfoques y aproximaciones teóricas que prosperan analizando el riesgo derivado de eventos tecnológicos; iniciativa que debe ser entendida como un intento por ahondar en (y aportar elementos de análisis a) el intrincado escenario de conflictividad social y ambiental contemporáneo.

\section{DEFINICIÓN DE RIESGO}

Si bien la antigüedad del concepto "riesgo" (bajo la idea de un "porvenir sin certeza") se ha hecho manifiesto $^{3}$, su definición resulta ser una tarea compleja, ya que ésta no sólo ha sufrido diferentes adaptaciones conceptuales en diferentes momentos de la historia, sino que cambia conforme a la disciplina y el enfoque desde el cual se le aborde. Por esto, y a pesar del tupido volumen de investigaciones, reflexiones y literatura al respecto, la mayor parte de los estudios concuerdan en afirmar que el origen de la palabra "riesgo" es desconocido y no cuenta con una definición homogénea, libre de cualquier problematización.

Desde una mirada terminológica, es prudente precisar que en español el término hace referencia a dos significados que, en inglés, corresponden a dos significantes diferentes: risk y hazard; los cuales se usan, algunas veces, de forma indistinta tanto en el lenguaje científico como, principalmente, en el lenguaje cotidiano. Lo que en inglés se denomina risk, en español equivale al término "riesgo", indicando con ello posibilidad -en el sentido de probabilidad- de daños o pérdidas. Pero a la vez, "riesgo" también se utiliza en español para designar la fuente de esos posibles daños (hazard en inglés), es decir, para denotar actividades, tecnologías, sustancias o acontecimientos capaces de producir afectaciones (Puy, 1995).

Kaplan y Garrick (1981), al abordar los aspectos cualitativos que atraviesan la noción de riesgo, puntualizan muy bien esta distinción entre hazard y risk. El primero es entendido como fuente de peligro, 
mientras el segundo como la posibilidad o el grado de probabilidad de daño. En este mismo sentido, en el Diccionario de la Real Academia Española (2001) se encuentran dos acepciones de "peligro": una como sinónimo de riesgo, en el sentido probabilístico ya referido, esto es: "riesgo o contingencia inminente de que suceda algún mal"; y otra poniendo más énfasis en la fuente de ese daño, esto es: "lugar, paso, obstáculo o situación en que aumenta la inminencia del daño".

Lo anterior parece sugerir que, cuando se pretenda precisar que se está haciendo alusión exclusivamente a un riesgo en el sentido de fuente de daño, parece más acertado utilizar palabras como "peligro", "amenaza" o "fuente de riesgo". Entretanto, la palabra "riesgo" se destinaría para enfatizar el significado probabilístico. No obstante, por ser "riesgo" la expresión de uso más común en la literatura, es el que mejor puede ser utilizado como término general cuando no sea necesaria la precisión entre ambos sentidos. Así se entenderá en el presente artículo.

Entretanto, desde una mirada académica no se ha podido establecer una definición unitaria del riesgo y menos todavía realizar una teoría coherente del mismo que permita estructurar los nuevos sectores de investigación y los múltiples resultados relativos a su problemática. Si bien la literatura sobre el riesgo proporciona una serie de clasificaciones del propio término, es posible identificar dos orientaciones generales desde el estudio académico. Por un lado, los enfoques de carácter positivista, en la línea de las ciencias naturales que trabajan con datos y hechos cuantificables. Por otro lado, aproximaciones de tipo interpretativo o hermenéutico que se centran en los aspectos más cualitativos del concepto.

\section{Enfoques estadístico-probabilísticos}

Trabajan con definiciones de cariz más formal, convencional, cuantitativo, matemático, donde el riesgo es entendido como una propiedad objetiva de un acontecimiento o una actividad, susceptible de medición probabilística a fin de definir sus efectos adversos. Desde este enfoque, "el riesgo hace referencia a las pérdidas esperadas y puede ser reducido a un valor numérico" (Hansson, 1989:107).

El origen de esta definición es posible encontrarlo en disciplinas como la ingeniería, la estadística, la física, la química, e incluso la economía, y responde a un "enfoque técnico" del término. Uno de sus objetivos es el de desarrollar una medida universalmente válida para el riesgo, por medio de la cual se pueda establecer comparaciones. Con ello, se confía en poder alcanzar una aclaración racional de la aceptabilidad de los distintos riesgos, conforme con el grado de su probabilidad y de sus consecuencias.

El núcleo de estas consideraciones lo constituye la fórmula:

\section{$\operatorname{Rie}|t=(A i, V e)| t$}

Esto significa que una vez conocida la amenaza o peligro Ai (entendida como la probabilidad de que se presente un suceso con una intensidad mayor o igual a i durante un período de exposición t) y conocida la vulnerabilidad Ve (entendida como la predisposición intrínseca de un elemento expuesto e a ser afectado o de ser susceptible a sufrir un daño ante la ocurrencia de un suceso con una intensidad i) el riesgo Rie se expresa como la probabilidad de que se presente una pérdida sobre el elemento e, como resultado de la ocurrencia de un suceso con una intensidad mayor o igual a $i$. Es decir, el riesgo en general puede entenderse como la probabilidad de pérdida durante un período de tiempo $t$ dado, resultado de la "convolución"4 de la amenaza y la vulnerabilidad (Cardona 2001:10).

Esta es la fórmula que define el llamado "riesgo objetivo" en la estimación de riesgo. En este sentido, el riesgo tiene que ver con la posibilidad de que sucedan acontecimientos futuros, combinada con la mayor o menor magnitud de las consecuencias de los mismos, esto es, con la magnitud de los daños potenciales. Así, la valoración del riesgo se reduce a una sola dimensión (el daño físico) resultado de la medición probabilística de un evento para definir los efectos adversos. La objetividad, en estos casos, estriba en reconocer el riesgo como algo "externo" a la percepción o a la interpretación particular social e individual, ya que al estar "ahí fuera", su efecto será efectivo independiente de algún elemento subjetivo.

Un ejemplo de tal posicionamiento puede reflejarse en lo dicho por Aquilino Morelle:

"El riesgo no tiene ninguna dimensión humana [...] De ahí que el riesgo sea una noción estadística, "universal" y "absoluta": universal, es decir que existe en todos los países donde se utiliza la técnica en 
cuestión; absoluta, o sea que presenta la misma incidencia, la misma posibilidad de realización en todos los países comparables en el plano científico y técnico" (Morelle, 1996, citado por Bourg; Schlegel, 2001:38).

Bajo una óptica general, la implicancia normativa consecuente de tal enfoque es clara:

"Ya que el daño físico es percibido como un efecto no deseado (al menos para la gran mayoría de la gente y para la sociedad en su conjunto), los análisis técnicos del riesgo pueden ser usados para revelar, evitar o modificar las causas que llevan a esos efectos no deseados" (Renn, 1992:59).

Sus funciones instrumentales en la sociedad están, por lo tanto, orientadas a corresponsabilizar y reducir riesgos mediante la mitigación de la presencia de daños, el establecimiento de estándares y las mejoras en la exactitud y/o veracidad y seguridad de los sistemas tecnológicos.

No obstante, para algunos autores:

"Una de las limitaciones más destacadas dentro de este enfoque es aquella relacionada con la unidad de medida utilizada en la detección de los daños [...] esta definición excluye acontecimientos inesperados y datos agregados sobre el espacio, las poblaciones y el tiempo. Los efectos indeseados son confinados al daño físico a los humanos y a los ecosistemas, excluyendo así los impactos sociales y culturales que aquellos puedan tener. Los análisis técnicos asumen además la forma de un espejo en relación entre la observación y la realidad y no consideran las causas del daño y la magnitud de las consecuencias que son mediadas a través de la experiencia social y de la interacción" (García, 2004:165-166).

En esta misma línea, López y Luján (2000:66-67) señalan que la fórmula es utilizable siempre que pueda determinarse la probabilidad del acontecimiento que produce el daño y la magnitud de éste de acuerdo con una medida cuantitativa. Esta es una limitación muy seria y bien conocida tanto al tratar de estimar la probabilidad (al llegar con frecuencia a los límites de lo que puede objetivamente conocerse) como al intentar determinar cuantitativamente la magnitud del daño (ya que no se ha podido desarrollar una medida unitaria y no problemática que dé cuenta de los distintos tipos de daño, pues su conversión en unidades monetarias, $u$ otro tipo de unidad económica, produce resultados arbitrarios muy discutidos) $)^{5}$.

\section{Enfoques socio-interpretativos}

Por otro lado, si bien la definición de riesgo cuenta con un relativo grado de consenso entre las disciplinas científicas naturales, desde las ciencias sociales no resulta tan fácil enunciar el riesgo como un concepto unidimensional y objetivo, ya que un mismo riesgo puede significar cosas distintas para diferentes personas o en diferentes contextos.

De esta forma, se puede abordar la definición de riesgo desde posturas más constructivistas que atiendan a todo aquello vinculado a su significado y a los aspectos personales, sociales y culturales que acompaña la definición de riesgo. Para algunos autores que han teorizado profundamente sobre la noción de riesgo (tales como Mary Douglas y Aaron Wildawsky) la idea de riesgo responde a "una construcción cultural situada entre las opiniones privadas subjetivas y el conocimiento científico público" (Douglas; Wildawsky, 1982:194). Desde esta óptica, el que un determinado riesgo sea provocado o no por decisiones humanas se relativiza, ya que la propia significación del riesgo -como realidad social construida que es- no es unívoca. Un riesgo que los expertos científicos consideran objetivamente producto de la naturaleza, otros individuos pueden verlo relacionado con decisiones humanas, y viceversa.

En este sentido, las nociones de riesgo no están basadas en razones prácticas o en juicios empíricos. Son nociones construidas socioculturalmente que enfatizan algunos aspectos del peligro e ignoran otros. Por tal razón, al momento de abordar la ciencia del riesgo o de llevar a cabo algún estudio en torno al mismo, no es posible partir de la idea de un sujeto individual libre de todo arraigo cultural, prescindiendo de las categorías de percepción presentes en la interacción social ${ }^{6}$. 
Así, de una u otra forma, se establecen socialmente las normas de aceptabilidad del riesgo, los principios codificadores por los que se reconocen los peligros y, como parte de la asignación de responsabilidades, las pautas culturales de qué constituye riesgos apropiados e inapropiados.

Se insiste, entonces, en el carácter multidimensional del riesgo, en el sentido que considera una serie de aspectos cualitativos que las personas toman en consideración a la hora de juzgar o evaluar un riesgo tecnológico (como el grado de voluntariedad a la exposición de un determinado evento, la inmediatez 0 demora de sus efectos, la credibilidad de las instituciones que lo gestionan, entre otros aspectos).

Dentro de esta perspectiva se encuentra el enfoque psicológico (centrando su atención en cuestiones tales como la explicación de por qué los individuos no siempre basan sus juicios sobre el riesgo en concordancia con lo expresado por los "expertos"; el estudio de los sesgos de la gente respeto a la regla de maximizar las utilidades y, en general, la importancia de las variables contextuales para dar forma a estimaciones individuales del riesgo) ${ }^{7}$; el enfoque antropológico (las aproximaciones al riesgo desde la antropología se basan, principalmente, en una perspectiva cultural. Suponen que los modelos culturales presentes en cada sociedad fuerzan a los individuos y a las instituciones a adoptar unos valores y a rechazar otros. Esto hace que los miembros de varias culturas identifiquen y seleccionen riesgos diferentes. El análisis cultural implica, pues, que la definición de efectos indeseados, la generación y la estimación de posibilidades, además de las construcciones de la realidad, dependen de la afiliación cultural del respectivo grupo social) ${ }^{8}$; y el enfoque sociológico (tienden a centrar su atención en todo tipo de acontecimientos indeseables que son definidos -e incluso construidos- socialmente. Asimismo, considera que las consecuencias reales de los mencionados acontecimientos están siempre mediadas por interpretaciones sociales y atadas a valores e intereses de grupo) ${ }^{9}$.

Las distintas aproximaciones a la definición de riesgo anteriormente explícitas comparten algunas consideraciones. En primer lugar, siguiendo las ideas de algunos autores (Yates, 1992; Puy, 1995), es posible asentir que el abordaje del riesgo se ve caracterizado por una distinción entre realidad y posibilidad, es decir, se considera cierta posibilidad de contingencia frente a un ulterior escenario. Si el futuro se considera predeterminado o independiente de las coetáneas actividades desarrolladas, el término riesgo no tendría sentido. Si el destino de una persona fuera predeterminado, no habría necesidad de anticipar resultados futuros, puesto que las consecuencias no podrían ser evitadas. Si se acepta la distinción entre realidad y posibilidad, el riesgo denota la posibilidad de que un estado de realidad indeseado pueda ocurrir como resultado, en este caso, de actividades humanas y eventos tecnológicos promovidos.

En segundo lugar, el riesgo es un concepto tanto descriptivo como normativo, puesto que permite establecer un modelo en el cual los seres humanos pueden hacer conexiones causales entre unas acciones y sus efectos. Y, además, supone que los efectos indeseados pueden ser eliminados o mitigados si los acontecimientos causales son evitados o modificados. Cavilar sobre qué constituye un riesgo y cuáles pueden ser sus consecuencias conlleva a identificar factores de riesgo, peligros, agentes generadores del riesgo, población expuesta, instituciones responsables de garantizar seguridad y, además, establecer unos parámetros que digan qué se debe hacer (o, como sucede más a menudo, qué no se debe hacer). Normalmente, suponer la posibilidad de un riesgo en una población implica la exigencia de reducirlo o eliminarlo.

En tercer lugar, otra idea subyacente a las diferentes perspectivas que abordan el riesgo es que la presencia del mismo genera consecuencias en términos de pérdidas o daños. Diferentes estudios han identificado diversas dimensiones susceptibles de tales alteraciones: el daño físico y biológico, la percepción del riesgo, el bienestar psicológico de los individuos, el impacto sobre el ambiente, entre otros. El riesgo, así, no se reduce a una sola magnitud, sino que cada una de estas dimensiones supone unas consecuencias distintas, a menudo, difícilmente conmensurables y equiparables entre sí.

Finalmente, y en cuarto lugar, se puede decir que las diferentes perspectivas sobre el riesgo están atravesadas por una incertidumbre intrínseca. Esta incertidumbre se presenta en varios ámbitos: respecto a los posibles escenarios donde pueden aparecer las pérdidas, lo cual implica que cuanto mayor sea esta incertidumbre mayor será el riesgo. También se puede hablar de incertidumbre sobre qué nivel de pérdidas ocurrirán, puesto que aunque se llegue a identificar las posibles dimensiones susceptibles de resultar afectadas, queda la incertidumbre sobre la probabilidad de que estas pérdidas sucedan o no (a mayor probabilidad mayor es el riesgo). Por último, también se habla de nivel de incertidumbre con respecto al grado de confianza de los parámetros estadísticos con los cuales se estima la probabilidad de ocurrencia de las pérdidas. 
Muchos de los estudios sobre riesgos, especialmente desde la perspectiva estadístico-probabilística, sólo contemplan la incertidumbre en el sentido de probabilidad de daños físicos, pero no en los otros aspectos mencionados (percepción del riesgo, bienestar psicológico de los individuos, costos y beneficios económicos, desconocimiento y temor, etcétera). Algunas experiencias reflejan que en realidad el espectro de daño es mucho más amplio y con repercusiones que no son consideradas en los análisis probabilísticos convencionales. Desde este punto de vista, la perspectiva numérica del análisis del riesgo resulta simple y limitada.

\section{ELEMENTOS DE LA NOCIÓN DE RIESGO}

Ciertamente el término "riesgo" no siempre formó parte del vocabulario de la sociedad o, por lo menos, no poseía el significado que posee actualmente. El hecho de que tal concepto adquiriera auge en las sociedades modernas tras la intensificación del avance tecnocientífico, no implica suponer que las sociedades premodernas (o algunas actualmente al margen de los procesos modernizadores de la sociedad occidental) no hayan disfrutado o padecido de sus avances tecnológicos, ni hayan sorteado los peligros y riesgos que los acompañan. El que algo suponga un "riesgo" en las sociedades denominadas postindustriales, reflexivas o avanzadas resulta de que las sociedades resuelven nombrar colectivamente un hecho como eso, como "riesgo" (García, 2004).

Rastreando algunas de las ideas expuestas por varios de los principales autores que han profundizado en la temática del riesgo, es posible reconocer un conjunto de conceptos que guardan concordancia, afinidad, cercanía o complementariedad con la noción de riesgo (Gráfico 1).

\section{Gráfico 1. Nociones vinculadas a la noción de riesgo}

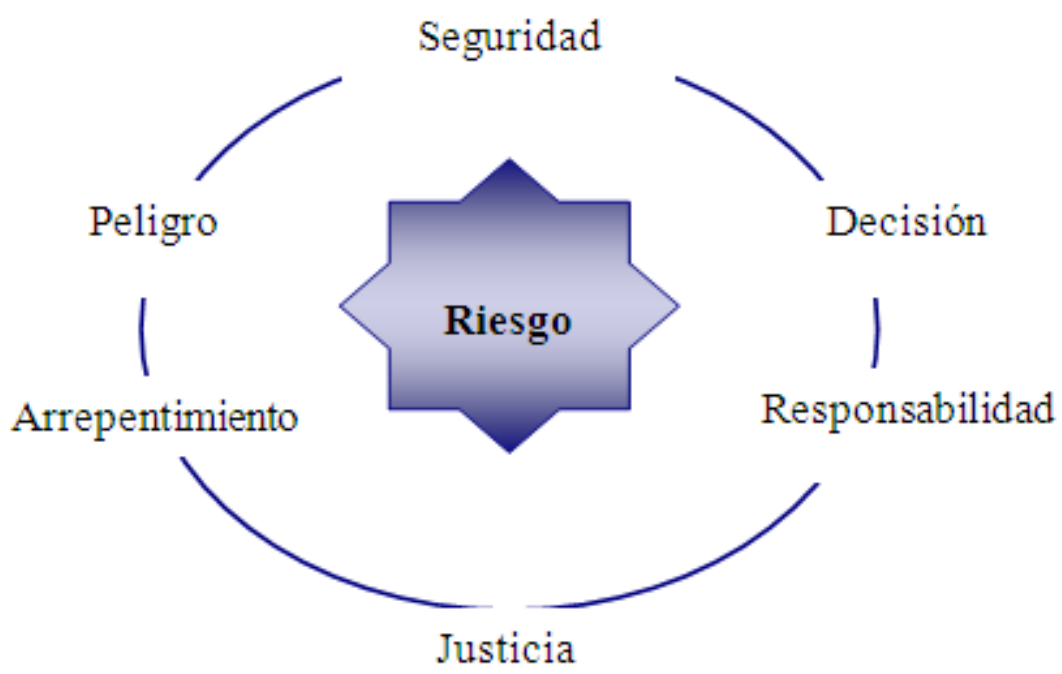

Fuente: Elaboración propia

Como señala Bechmann (1995:68), las nociones de riesgo y seguridad son complementarias: donde hay inseguridad, ésta trata de ser controlada mediante el cálculo del riesgo. Partiendo de la idea de que la inseguridad logra darse por la presencia de un peligro o una amenaza, el hecho de expresar tales eventos generadores de inseguridad en términos de riesgo, da a entender que la sociedad puede llevar a cabo acciones o intervenciones para manejarlos, controlarlos, minimizarlos o, por lo menos, hacer algo al respecto: recoger más información, invertir más recursos en tiempo o dinero, promulgar nuevas leyes, entre otras iniciativas.

Tras la relación entre las nociones de peligro y riesgo se derivan esfuerzos por "conocerlo", por identificarlo y caracterizarlo. En otras palabras, por convertirlo en un objeto de estudio de un conjunto de 
acciones técnicamente más o menos controladas y eficaces. Esto, inevitablemente, demanda la necesaria tarea de tomar decisiones frente a diferentes alternativas de elección, ya que "sólo podemos hablar de riesgos en la medida que las consecuencias pueden atribuirse a las decisiones" (Beriain, 1999:230. La negrita es suya). Empero, aunque al momento de decidir entre múltiples alternativas la opción supuestamente segura no necesariamente es la más beneficiosa, su elección puede representar la pérdida de oportunidades. De este modo, a decir de López y Luján (2000:23), "se establece una relación entre los conceptos de riesgo y arrepentimiento: el cálculo del riesgo es un modo de controlar la inseguridad, pero también de minimizar el arrepentimiento" (la cursiva es suya).

De acuerdo a lo anterior, el riesgo está íntimamente ligado a una decisión -social o individualmentetomada. El origen del riesgo (y por la tanto de las ulteriores alteraciones desencadenas) ya no reside en una voluntad externa o independiente, por el contrario, la responsabilidad de tal panorama es atribuido a decisiones tomadas y a elecciones preferidas.

Concordando con algunos planteamientos de la sociedad del riesgo del sociólogo alemán Ulrick Beck, la cuestión no es tanto si los peligros tecnocientíficos contemporáneos son mayores o menores que los del pasado, sino que hoy los peligros son habitualmente imputados a acciones y decisiones humanas, y por lo tanto, se les otorga la forma de riesgos. Sin embargo, no hay que olvidar que los riesgos hoy en día son utilizados como instrumento de distribución de cagas sociales y responsabilidades políticas, y que la (in)justicia es una constante en la distribución de estos efectos colaterales, de estos "males", de estas situaciones no deseadas resultado de sortear una elección en particular. No está de más recordar que "la actual distribución de riesgos refleja sólo la vigente distribución de poder y posición social, el tema de la justicia suscita cuestiones políticas fundamentales" (Douglas, 1996:32).

Esto último ha llevado a discusiones sobre aquellos riesgos que se corren sin haber entrado en un proceso de toma de decisión, bien porque no han sido percibidos o bien porque, aún al hacerlo, no han sido reconocidos como objetos sobre los que cabe decidir. Por ello, de forma acertada, Starr (1969) diferenció entre "riesgos asumidos" y "riesgos impuestos" por otros, ofreciendo importantes herramientas en el debate de la aceptabilidad del riesgo ${ }^{10}$.

Ahora bien, estos planteamientos en torno al riesgo han generado el surgimiento de diferentes discusiones sobre la forma de conceptualizar y sobre el mejor modo de analizar el riesgo. En este sentido, diferentes autores han propuesto variados esquemas de clasificación del concepto de riesgo, donde el grado de valoración presente en la estimación del mismo actúa como un elemento significativo de diferenciación (Cuadro 1). 
Cuadro 1. Clasificaciones del concepto de riesgo

\begin{tabular}{|c|c|c|c|}
\hline Clasificación & Aspecto & Descripción & Autor \\
\hline $\begin{array}{l}\text { Positivistas } \\
\text { /Relativista }\end{array}$ & $\begin{array}{l}\text { Posiciones respecto } \\
\text { al riesgo (fiabilidad } \\
\text { epistemológica) }\end{array}$ & $\begin{array}{l}\text { Los positivistas piensan que es posible } \\
\text { conseguir estimaciones totalmente objetivas } \\
\text { del riesgo, mientras los relativistas niegan tal } \\
\text { posibilidad. }\end{array}$ & $\begin{array}{l}\text { Shrader- } \\
\text { Frechette } \\
\text { (1991) }\end{array}$ \\
\hline $\begin{array}{l}\text { Sociológicos y } \\
\text { Metacientíficos / } \\
\text { positivistas }\end{array}$ & Tipos de análisis & $\begin{array}{l}\text { Por un lado se afirma que es posible analizar } \\
\text { los enunciados de riesgo teniendo en cuenta } \\
\text { la influencia de los valores tanto desde un } \\
\text { punto de vista sociológico como } \\
\text { metacientífico. En el otro extremo se } \\
\text { encontraría un punto de vista positivista según } \\
\text { el cual los valores no influyen en la } \\
\text { determinación del riesgo. }\end{array}$ & $\begin{array}{l}\text { Mayo } \\
(1991)\end{array}$ \\
\hline $\begin{array}{l}\text { Probabilista / } \\
\text { Contextualistas }\end{array}$ & $\begin{array}{l}\text { Contenidos de las } \\
\text { afirmaciones }\end{array}$ & $\begin{array}{l}\text { Para los probabilistas los enunciados de } \\
\text { riesgos se refieren a probabilidades y para los } \\
\text { contextualistas se refieren a otros factores } \\
\text { como la justicia social, por ejemplo. }\end{array}$ & $\begin{array}{l}\text { Thomson; } \\
\text { Dean } \\
\text { (1996) }\end{array}$ \\
\hline $\begin{array}{l}\text { Ecológico / } \\
\text { Cultural }\end{array}$ & Tipo de enfoque & $\begin{array}{l}\text { Las nociones de riesgo no están basadas en } \\
\text { razones prácticas o en juicios empíricos } \\
\text { (como lo afirma el enfoque ecológico). Son } \\
\text { nociones construidas culturalmente que } \\
\text { enfatizan algunos aspectos del peligro e } \\
\text { ignoran otros. }\end{array}$ & $\begin{array}{l}\text { Douglas } \\
\text { (1996) }\end{array}$ \\
\hline $\begin{array}{l}\text { Material / } \\
\text { Inmaterial }\end{array}$ & $\begin{array}{l}\text { Mediación de la } \\
\text { conceptualización }\end{array}$ & $\begin{array}{l}\text { La inmaterialidad se asienta en que "no hay } \\
\text { más que sociedad" en la mediación de la } \\
\text { conceptualización del riesgo. Mientras que la } \\
\text { materialidad reconoce un "producto de la } \\
\text { acción", una amenaza existente necesaria de } \\
\text { considerar. }\end{array}$ & $\begin{array}{l}\text { Beck } \\
(2002)\end{array}$ \\
\hline
\end{tabular}

Fuente: Elaboración propia basada en la bibliografía citada.

Como puede deducirse de tal esquema, por lo menos hay dos elementos conflictivos involucrados en el proceso de conceptualización del riesgo: uno, las controversias sociales que atraviesan y enmarcan su propia definición y, dos, el papel que el conocimiento científico desempeña. A pesar de ser diferenciados como dos instancias que responden a reflexiones particulares, su línea de separación no es del todo clara y se encuentran relacionadas. Por ejemplo, para positivistas, probabilistas y el enfoque ecológico y material, la problemática social gravita en torno a la determinación de la cuantificación del riesgo, otorgando a la ciencia y al conocimiento experto la potestad de pronunciarse como la máxima instancia al respecto. En cambio, para relativistas, contextualistas y el enfoque cultural e inmaterial, la controversia social desborda la mera cuantificación y resalta los principios éticos y políticos, al igual que el conjunto de relaciones sociales y culturales que atraviesan la conceptualización del riesgo. De esta forma, se afirma que el conocimiento científico puede ayudar a aportar elementos de discusión y análisis, pero no resulta por sí mismo globalmente aceptable, ni totalmente satisfactorio para llevar a cabo una efectiva gestión del riesgo.

Así, la noción de riesgo ha sufrido una especie de "metamorfosis semántica" (García, 2004), es decir, cambios en la manera de formularlo y significarlo. Ello, entre otras cosas, es el resultado de las históricas y variables configuraciones sociales que, en su desarrollo, elaboran distintos medios y herramientas para entender, manejar, gestionar y vivenciar el riesgo.

\section{CONTEMPORANEIDAD DEL RIESGO TECNOLÓGICO}

El riesgo tecnológico, entendido como un elemento constitutivo del amplio conjunto de consideraciones inevitablemente ligadas al devenir científico-tecnológico, emerge como un síndrome de advertencia, de reflexión sobre el nivel de incidencia de la tecnología en las sociedades modernas. Estas últimas, susceptibles de ser interpretadas como "sociedades del riesgo" (Beck, 1998, 2002), se encuentran fuertemente caracterizadas por la proliferación de situaciones socialmente conflictivas derivadas tanto del progreso tecnocientífico, como de la propia complejidad de la organización social. 
Algunos autores concuerdan en afirmar que el impulso de la investigación contemporánea del riesgo de procesos tecnológicos, en un sentido estricto, estuvo íntimamente relacionada con el desarrollo forzado de la energía nuclear a comienzos de los años sesenta y con el conjunto de debates asociados (Rodríguez, 1999; López; Luján, 2000). Desde los años setenta emergió una ola sin precedentes de gran interés público y académico por el estudio del riesgo, lo cual se tradujo en la aparición de numerosas revistas, organizaciones de sociedades profesionales, celebración de congresos, desarrollo de cursos especializados, etcétera, constituyéndose el estudio del riesgo es un importante campo multidisciplinar de investigación y reflexión académica.

Vista desde una perspectiva amplia, la tecnología ha sido una fuerza poderosa en el desarrollo de la civilización occidental, más aún, cuando se ha fraguado su vínculo con la ciencia. Aumentando las posibilidades de incidir y alterar el mundo, el ser humano se ha servido de la tecnología para acondicionar su entorno a fin de que se adapte mejor a sus necesidades. No obstante, algunos de los resultados de tales intervenciones son con frecuencia confusos e impredecibles, llegando a incluir no sólo beneficios o costos económicos a mediano y largo plazo, sino también, y especialmente, riesgos con la capacidad de afectar a diferentes grupos sociales en distintos momentos ${ }^{11}$.

El riesgo, en este sentido, se ha convertido en la noción clave sobre la que pivotan gran parte de los diagnósticos sociales (sean estos económicos, políticos, técnicos, jurídicos o sociológicos), pasando a ocupar tal concepto un lugar relevante dentro de los debates contemporáneos. Gracias a ello, el desarrollo tecnocientífico de las sociedades modernas ha puesto en evidencia la presencia de riesgos que van más allá de los naturales conocidos hasta ahora por la humanidad. A los clásicos riesgos ligados a los elementos naturales tales como inundaciones, incendios, sequías, etcétera, se agregan en la actualidad aquellos que son producto "exclusivo" de la actividad humana, tales como la energía nuclear, las ondas electromagnéticas, la ingeniería genética, la informática, la nanotecnología, los numerosos procesos basados en la utilización de compuestos químicos y, como parte de estos últimos, la amplia e intensiva utilización de plaguicidas en sistemas agrícolas, entre otros.

Hacer frente a los llamados riesgos tecnológicos de tipo antropogénico ${ }^{12}$ permite reconocer cómo de forma simultánea al avance del conocimiento científico se extiende, paradójicamente, enormes vacíos cognitivos y profundas lagunas de ignorancia e incertidumbre. Si bien a la luz de una multitud de variables es posible admitir que cuantiosas innovaciones tecnológicas han contribuido efectivamente al mejoramiento de un sinnúmero de circunstancias desfavorables (motivando un papel activo de la sociedad para enfrentar situaciones adversas), también es posible asentir que a la sombra de tales procesos se generan iniciativas nocivas, riesgosas e inciertas con explícitos y potenciales efectos sobre la sociedad. En palabras de López y Luján:

"Es el nuevo mundo del riesgo asociado a la ciencia y la tecnología actual: cuanto más conocemos los riesgos, mejor apreciamos la gran extensión de nuestra ignorancia; cuanto más hacemos por controlarlos, mayores son los riesgos generados en otra parte del sistema" (2000:13).

De esta forma, el propio decurso tecnológico parece avanzar en medio de una conflictiva relación formulada en términos de "afecto/rechazo": "afecto", al avizorar la sociedad el amplio abanico de posibilidades inimaginables emergidas tras su vertiginoso avance, y al ofrecer ésta (la tecnología) soluciones temporales a calamidades expuestas. Y "rechazo", al estimular la aparición de un conglomerado de situaciones confusas y amenazantes, y al suscitar peligros y situaciones no deseadas como parte de este mismo proceso. Tal relación es enunciada por el filósofo alemán Nicholas Rescher de la siguiente manera:

"Por una parte, sólo ella [la tecnología] es capaz de proporcionarnos los requisitos para hacer posible la vida humana dentro de las condiciones del mundo moderno. Por otra parte, la tecnología misma hace que, de muchas maneras, la vida sea más complicada, menos agradable y más peligrosa" (Rescher, 1999:46)

Ahora bien, ¿cuáles son esas particulares circunstancias que permiten afirmar la existencia de un estado de "novedad" tecnológico? ¿Qué condiciones permiten asentir la vivencialidad de unas exclusivas condiciones de riesgo en relación a otro contexto anteriormente enfrentado?

Algunos autores identifican un escenario histórico concreto caracterizado, en primer lugar, por el nivel de los impactos generados sobre la biosfera por parte de los sistemas industriales en las sociedades modernas, llegando a hablar de "daños catastróficos e irreversibles para los seres vivos y los 
ecosistemas". En segundo lugar, los procesos de toma de decisiones, aunque están basados en el mejor conocimiento científico disponible, a menudo se desarrollan "en condiciones de ignorancia o incertidumbre" (Riechmann, 2002:9). Y, en tercer lugar, estas "nuevas" problemáticas enfrentadas resultan ser, además de globales en escala y de larga duración en sus impactos, complejas, novedosas y variables; constituyéndose, por lo tanto, en fenómenos poco comprendidos en su totalidad (Funtowicz; Ravetz, 1993).

Al respecto, Ulrick Beck reconoce que la sociedad industrial actúa como un referente histórico de cambio donde, más allá de implicar procesos de transformación en distintas instancias de orden económico, productivo, social, ecológico, cultural, entre otros, es posible entrever la aparición de amenazas cada vez más extensas y complejas inherentes al propio avance de los sistemas industriales. Es decir, este autor reconoce una relación cercana entre el avance industrial, científico y, por lo tanto, tecnológico, con la aparición de ciertos riesgos que, en caso de materializarse, imprimirían su capacidad de transformación y afectación. Ante esto, aquello que en un principio podría verse como efectos secundarios del desarrollo, en la actualidad reclama un estatuto de igualdad con los efectos positivos. En otros términos, reclama convertirse en variables endógenas del modelo de desarrollo.

Además, el autor en cuestión hace hincapié en que los riesgos tecnológicos que atraviesan la sociedad tienen un origen ya no externo, ajeno, extrahumano, sino interno, y son provocados por la capacidad del hombre para autoconfigurarse y auto-transformarse:

"A diferencia de todas las épocas anteriores (incluida la sociedad industrial) la sociedad del riesgo se caracteriza esencialmente por una carencia: la imposibilidad de prever externamente las situaciones de peligro. A diferencia de todas las anteriores culturas y de todas las fases de desarrollo social, que se enfrentaron de diversos modos con amenazas, la actual sociedad se encuentra confrontada consigo misma con relación a los riesgos. Los riesgos son el producto histórico, la imagen refleja de las acciones humanas y de sus omisiones, son la expresión del gran desarrollo de las fuerzas productivas" (Beck, 1998:237. La negrita es suya).

De alguna forma, tal como fue mencionado anteriormente, los riesgos existentes en las sociedades preindustriales podían ser atribuidos o descargados sobre "fuerzas externas", llegando a acusar a "otro" por engendrar alguna situación problemática con repercusiones sociales ${ }^{13}$.

De este modo, con la aparición y extensión de la tecnología a todos los ámbitos de la vida, nace para la sociedad un potencial de riesgo tecnológico (en parte) desconocido por su magnitud (al no conocer con certeza el nivel de incidencia que pueda tener) y por su alcance (al desconocer el límite espacial y temporal del mismo). Como resultado, siguiendo algunas ideas de Luhmann (1996:123), la noción de riesgo no sólo está hoy en el centro de las polémicas públicas, sino también, en el núcleo del debate de las especialidades científicas más diversas e, inclusive, en las ciencias más variadas.

\section{BIBLIOGRAFÍA}

- $\quad$ BECHMANN, Gotthard (1995): "Riesgo y desarrollo técnico-científico. Sobre la importancia social de la investigación y valoración del riesgo", Cuadernos de Sección, Ciencias Sociales y Económicas, n. 2, pp.59-98.

- $\quad$ BECK, Ulrich (1998): La sociedad del riesgo. Hacia una nueva modernidad, Barcelona, Paidos.

- $\quad$ BECK, Ulrich (2002): La sociedad del riesgo global, Madrid, Siglo XXI.

- BERIAIN, Josetxo (1999): "Genealogía sociológica de la contingencia: del destino dado metasocialmente al destino producido socialmente", en Ramos, R; García Selgas, F. Globalización, Riesgo, Reflexividad. Tres temas de Teoría Social Contemporánea, Madrid, Centro de Investigaciones Sociológicas (CIS), pp. 205-248.

- BOURG, Dominique; SCHLEGEL, Jean-Louis (2001): Parer aux risques de demain. Le principe de précaution, Paris, Seuil.

- CARDONA, Omar (2001): Estimación holística del riesgo sísmico utilizando sistemas dinámicos complejos, Tesis doctoral, Barcelona, Universitat Politècnica de Catalunya.

- CARRIZOSA, Julio (2001): ¿Qué es ambientalismo? La visión ambiental compleja, Bogotá, PNUMA / IDEA / CEREC. 
- COVELLO, Vincent; MUMPOWER, Jeryl (1985): "Risk Analysis and Risk Management: An historical perspective", en Risk Analysis, Society for Risk Analysis, v. 5, n.2, pp. 103-120.

- DOUGLAS, Mary (1996): La aceptabilidad del riesgo según las ciencias sociales, Barcelona, Paidos.

- DOUGLAS, Mary; WILDAVSKY, Aaron (1982): Risk and Culture: An essay on the selection of technological and environmental dangers, Berkeley, University of California Press.

- ECHEVERRÍA, Javier (2001): "Tecnociencia y sistema de valores", en López, J.; Sánchez, J. (eds.): Ciencia, tecnología, sociedad y cultura en el cambio de siglo, Madrid, Biblioteca Nueva/OEI.

- ESPLUGA, Josep (2004): "Conflictes socioambientals i estudi de la percepció social del risc", en Papers. Revista de sociología, n. 72, Barcelona, Universitat Autònoma de Barcelona, pp. 145162.

- FUNTOWICZ, Silvio; RAVETZ, Jerome (1993): Epistemología política. Ciencia con la gente, Buenos Aires, Centro Editor de América Latina.

- GALLOPíN, Gilberto et al (2001): "Una ciencia para el siglo XXI: del contrato social al núcleo científico", en Revista internacional de ciencias sociales. La ciencia y sus culturas, n. 168, pp. 4762.

- $\quad$ GARCÍA, Anna (2004): Negociar el riesgo. Una propuesta para la gestión de riesgos en sistemas tecnológicos complejos, Tesis doctoral, Barcelona, Universitat Autònoma de Barcelona.

- $\quad$ HANSSON, Sven (1989): "Dimensions of risk", en Risk Analisys, v. 9, pp. 107-112.

- JOHNSTON, Paul; SANTILLO, David; STRINGER, Ruth (1996): "Risk assessment and reality: Recognizing the limitations", en Quint, M.; Purchase, R; Taylor, D. (eds.): Environmental Impact of Chemicals: Assessment and Control. Cambridge, Royal Society of Chemistry, pp. 223-239.

- KAPLAN, Stanley; GARRICK, B. (1981): "On the quantitative definition of risk", en Risk Analisys, v. 1, n. 1, pp. 11-27.

- LACKEY, Robert (1997): "Ecological risk assessment: Use, abuse, and alternatives", en Environmental Management, v. 21, n. 6, pp. 808-812.

- LAVELL, Allan (2003): La gestión local del riesgo nociones y precisiones en torno al concepto y la práctica, Panamá, CEPREDENAC - PNUD.

- $\quad$ LÓPEZ, José; LUJAN José (2000): Ciencia y política del riesgo, Madrid, Alianza Editorial.

- LUHMANN, Niklas (1996): "El concepto de riesgo", en Beriain, J. (comp.): Las consecuencias perversas de la modernidad. Modernidad, contingencia y riesgo, Barcelona, Anthropos, pp. 123153.

- MAYO, Deborah (1991): "Sociological versus metascientific views of Risk Assessment”, en Mayo, D.; Hollander, R. (eds.): Acceptable evidence: Science and values in Risk Management, Oxford, Oxford University Press.

- MOLAK, Vlasta (1997) (ed.): Fundamentals of Risk Analysis and Risk Management, New York, Lewis Publishers.

- $\quad$ PUY, Ana (1995): Percepción social de los riesgos, Madrid, Mapfre.

- RAMíREZ, Omar (2006): "Reflexiones sobre el monocultivo de soya transgénica en Argentina: Una aproximación desde la geografía del azar tecnológico", en Revista Gestión y Ambiente, v. 9, n. 3, pp. 81-90.

- RENN, Ortwin (1992): "Concepts of Risk", en Krimsky, Sh.; Golding, D. (eds.): Social Theories of Risk, Westport, Praeger Publishers.

- RESCHER, Nicholas (1999): Razón y valores en la Era científico-tecnológica, Barcelona, Paidos.

- RIECHMANN, Jorge (2002): "Introducción: un principio para reorientar las relaciones de la humanidad con la biosfera", en Riechmann, J.; Tickner, J. (coords.): El principio de precaución. En medio ambiente y salud pública: de las definiciones a la práctica, Barcelona, Icaria.

- RODRíguEZ, J. (1999): "El riesgo como utopía negativa. Notas para una reflexión", en Ramos, R; García Selgas, F. Globalización, Riesgo, Reflexividad. Tres temas de teoría social contemporánea, Madrid. Centro de Investigaciones Sociológicas (CIS), pp. 191-204.

- $\quad$ SHRADER-FRECHETTE, Kristin (1991): Risk and rationality. Philosophical foundations for populist reforms, Berkeley, University of California Press.

- STARR, Chauncey (1969): "Social benefit versus technological risk", en Science, n. 165, pp. 1232-1238.

- THOMSON, Paul.; DEAN, Wesley (1996): "Competing conceptions of risk" [en línea]. En: Internet <http://www.piercelaw.edu/risk/vol7/fall/thompson.htm> (consulta 06 de febrero de 2009).

- $\quad$ YATES, Frank (ed.) (1992): Risk-Taking Behaviour, Chinchester, UK, John Wiley \& Sons. 
1. MSc. Sistemas Ambientales Humanos del Centro de Estudios Interdisciplinarios (CEI) de la Universidad Nacional de Rosario (Argentina). Actualmente vinculado a la Escuela de Ciencias Agrícolas, Pecuarias y del Medio Ambiente - ECAPMA de la Universidad Nacional Abierta y a Distancia - UNAD (Colombia). E-mail: omarjrh@yahoo.com.

2. Para este caso, se interpreta la relación ciencia y tecnología en términos de "tecnociencia". Es decir, como un complejo entramado donde, por un lado, la ciencia y la tecnología se hayan fuertemente imbricadas en una relación de feedback mutuo: la ciencia permite el desarrollo de nuevas tecnologías, que a su vez aceleran o influyen el proceso científico, que a su vez permite nuevas tecnologías. A decir de Echeverría (2001:222), "no hay progreso científico sin avance tecnológico, y recíprocamente".

3. Según varios autores, el concepto de riesgo tuvo orígenes en las antiguas ideas religiosas relacionadas con la posibilidad de algo después de la vida. Este es el caso del Fedón de Platón (Siglo IV a.C.) y de otros escritos que trataron sobre la inmortalidad del alma y lo que le podría ocurrir después de la vida, según como cada persona conduce su aquí y ahora (Covello; Mumpower, 1985). Además, siguiendo a Molak (1997:3-4), los antiguos griegos y romanos ya habían observado correlaciones entre enfermedades y exposiciones ambientales. Por ejemplo, Hipócrates (siglo IV a.C.) correlacionó la aparición de enfermedades con exposiciones ambientales, Vitruvio (siglo I a.C.) dio cuenta de la toxicidad del plomo y Agrícola (siglo XVI d.C.) dio cuenta de la correlación entre la exposición ocupacional a la minería y la salud.

4. La convolución es un concepto que se refiere a la concomitancia y mutuo condicionamiento, en este caso, de la amenaza y la vulnerabilidad. Dicho de otra forma, y bajo esta perspectiva, no se puede ser vulnerable si no se está amenazado y no existe una condición de amenaza para un elemento, sujeto o sistema si no está expuesto y es vulnerable a la acción potencial que representa dicha amenaza. En otras palabras, no existe amenaza o vulnerabilidad independientemente, pues son situaciones mutuamente condicionantes que se definen en forma conceptual de manera independiente para efectos metodológicos y para una mejor comprensión del riesgo (Cardona 2001:10-11).

5. Para una crítica general del enfoque técnico, ver: Douglas (1996), Johnston; Santillo; Stringer (1996), Lackey (1997), entre otros. Estos autores señalan, entre otras cosas, la estrechez del esquema de análisis propuesto al centrarse en un único criterio para la identificación, evaluación y gestión de los riesgos. Ello no significa, no obstante, que dichos análisis sean innecesarios o menos relevantes que otros conceptos de riesgo.

6. Tal como afirma William Torry: "Lo que los individuos hacen o dicen que hacen en cuanto a proteger la seguridad de su mundo depende de un gran número de factores que incluirán su posición social, nivel de formación cultural, acceso a fuentes de crédito tales como las insertas en redes de parentesco, habilidad técnica, tamaño y diversidad de propiedades, opciones de empleo, exigencias de trabajo doméstico, pertenencia a organizaciones voluntarias, capacidad productiva del capital y compromisos con valores culturales y con conveniencias religiosas" (Torry, 1979, citado por Douglas, 1996:51).

7. Como afirman López y Luján (2000:69) "asumiendo una especie de dualismo de propiedades (pues suele reconocerse también la realidad objetiva del riesgo, en los términos del enfoque técnico), aquí el riesgo es entendido en términos de representación cognitiva, como un estado mental de agentes individuales ("riesgo percibido"), con probabilidades subjetivas y grados de aceptabilidad (determinables, por ejemplo, a través de escalas de preferencias, y sistematizables a través de técnicas de factorización) que dependen de una serie de variables contextuales vinculados a estados de creencia o disposiciones comportamentales del agente".

8. Douglas y Wildavsky (1982), partiendo de la Teoria Cultural, suponen que las creencias y valores compartidos por determinados grupos (sociales y culturales) influyen en la selección de lo que se considera o no como un riesgo, de tal manera que las personas de estos grupos se preocupan especialmente de aquellos acontecimientos o aspectos que más pueden afectar o poner en peligro sus sistemas de creencias o valores, su manera de entender y de vivir las relaciones sociales. Desde esta perspectiva, cada grupo social selecciona (inadvertidamente) los riesgos que "quiere" temer con la finalidad de dar coherencia a su forma de vivir y a sus propios valores e ignora el resto de los posibles riesgos (que sí pueden ser relevantes para otros grupos sociales). 
9. En este enfoque, "los riesgos no son vistos ni como propiedades objetivas que depende de cómo sea físicamente el mundo, ni como propiedades subjetivas que dependen de cómo sean cognitivamente los individuos". Por el contrario, los riesgos son vistos como "construcciones sociales que dependen de factores socioculturales vinculados a estructuras sociales dadas" (López; Luján, 2000:72). Si bien no son construcciones gratuitas, éstas son concordantes dentro de las estructuras sociales en que operan. En este sentido, no se contempla la aceptación o no de riesgos tecnológicos como resultado de una decisión individual subjetiva, sino, por el contrario, "el enfoque es contextualizado: se centra en el estudio de los factores que hacen que determinados puntos de vista respecto a riesgos resulten dominantes en grupos sociales dados, o bien que se produzcan polarizaciones y enfrentamientos respecto a la distribución del riesgo" (López; Luján, 2000:73).

10. En este mismo sentido, Luhmann diferencia entre los conceptos de "riesgo" y "peligro": "El daño eventual es visto como consecuencia de la decisión, por lo cual se habla de riesgo de la decisión. Hablamos de peligro cuando el hipotético daño, entendido como causado desde el exterior, se le atribuye al entorno" (1996:144). Es decir, el riesgo sería el resultado de decisiones propias, mientras el peligro deriva de imposiciones o de decisiones ajenas.

11. "Cada salto tecnológico ha estado acompañado por tres procesos íntimamente relacionados entre sí: el primero, la identificación de ciertas condiciones valoradas como "problemáticas" o "susceptibles de ser mejoradas" (hambre, pobreza, enfermedades, entre otras). El segundo, la aparición de sistemas tecnológicos que, a la luz de la promesa de resolver dichas condiciones, fomentan una representación "benéfica" de la tecnología. Empero, a la sombra de tal proceso, derivan otras situaciones conflictivas e inciertas, estimulando una imagen riesgosa de la misma. El tercero, inmerso en una lógica circular o espiral, es la tendencia a realizar innovadores saltos tecnológicos que puedan resolver, de vuelta, los problemas anteriormente provocados (Ramírez, 2006:82).

12. Según Lavell (2003:18), es posible identificar factores de riesgo naturales (derivados de la misma dinámica de la naturaleza, dentro de los cuales se encuentran fenómenos como sismos, huracanes, tsunamis y erupciones volcánicas) y antropogénicos (derivados por completo de acciones humanas).

13. En este sentido, varios autores han reconocido la gran influencia de la religión como una importante fuente inductiva de la atribución causal de situaciones riesgosas a fuerzas externas, alejadas de cualquier responsabilidad humana. Esta idea es expuesta por Cardona (2001:7) de la siguiente manera: "los fenómenos naturales, las pestes y otras crisis sociales también fueron relacionadas con designios divinos o mala suerte. Durante siglos este tipo de creencias se mantuvo en una u otra forma dentro de las sociedades, particularmente por la influencia de la religión". Entretanto, Beck (2002:78) afirma que los riesgos preindustriales respondían a "innumerables acusaciones, pero se dirigían contra los dioses o contra Dios, tenían una "motivación religiosa", por expresarlo de forma simple, y carecían de carga política, al contrario de lo que ocurre con los riesgos industriales". 